\title{
Third Asian Conference on Mechanics of Functional Materials and Structures (ACMFMS 2012)
}

Received: 12 June 2014 / Published online: 31 July 2014

(C) Springer-Verlag Wien 2014

This special issue comprises of selected papers that were presented in the Third Asian Conference on Mechanics of Functional Materials and Structures (ACMFMS 2012), organized by the Indian Institute of Technology Delhi in New Delhi, India, during December 5-8, 2012. The ACMFMS series began in 2008 with the aim of providing a forum for researchers and academics, particularly from East Asia and the neighboring region, to present their latest findings, exchange ideas, and establish relationships for future cooperation and subsequent promotion of research in the theoretical and applied mechanics of solids. The first in the series was held in Matsue, Japan in October, 2008 and the second in Nanjing, China, in October, 2010.

In recent years, solid mechanics has emerged as one of the key areas of engineering science, controlling technological developments in many areas ranging from nanotechnology to space technology. It offers fundamental understanding of materials, structures, and diverse complex systems such as those found in biology and nanotechnology, and many physical phenomena at multiple scales. The concept of multifunctional materials and structures has attracted multifaceted research activities in applications spanning over mechanical, aerospace, civil, materials, biomedical, naval, and arctic engineering. The papers presented in this issue represent the recent developments in the mechanics of functional materials and structures, as well as its applications to modern technology.

ACMFMS 2012 received 359 submissions from 13 countries, reflecting the growing interest in the conference. Among the accepted submissions, 239 four-page papers have been published in the Proceedings of the conference, covering mechanics of functional and smart structures, mechanics of functional materials, structural health monitoring, elasticity and thermo-elasticity, mechanics of composite and functionally graded materials and structures, bio- and nano-mechanics, dynamics and stability, impact, plasticity and contact, fatigue and fracture, and solid-fluid interaction. With more than 250 delegates, 235 presentations, seven keynote lectures, and eight invited lectures, ACMFMS 2012 was a great success.

Based on two reviews of the four-page papers, only a selected few of 22 were invited to submit the fulllength manuscripts. The full-length submissions were then reviewed by two or more referees in the subject area drawn from across the globe. Finally, 17 have made it through the rigorous review process and have been accepted for this special issue.

I would like to express my heartfelt gratitude to the Editorial Board of the ACTA MECHANICA headed by Prof. Hans Irschik for dedicating this precious volume for ACMFMS 2012, and bestowing the trust on me for editing it. I also extend my sincere thanks to all the authors for their fine contributions and to the large number of reviewers for sparing their valuable time in providing sincere and critical reviews. I hope and trust that the ACMFMS community will grow stronger with each conference. The next ACMFMS-2014 will be held in the beautiful city of Nara, Japan, during October 10-13, 2014, and I expect a strong attendance.

S. Kapuria $(\varangle)$

Department of Applied Mechanics, Indian Institute of Technology Delhi, Hauz Khas, New Delhi, India

E-mail: kapuria@am.iitd.ac.in

Tel.:+91-11 26591218 
The organizing committees for ACMFMS2012 are listed below.

\section{Chair}

Chair: Santosh Kapuria (Indian Institute of Technology Delhi, India)

Co-Chair: Sei Ueda (Osaka Institute of Technology, Japan)

Secretary: S. Pradyumna (Indian Institute of Technology Delhi, India)

\section{International advisory committee}

C. K. Chao (National Taiwan University of Sci and Tech, Taiwan)

T. K. Datta (Indian Institute of Technology Delhi, India)

M. R. Eslami (Amirkabir University of Technology, Iran)

D.-N. Fang (Peking University, China)

T. Furukawa (University of Ryukyus, Japan)

N. K. Gupta (Indian Institute of Technology Delhi, India)

N. Noda (Shizuoka University, Japan)

Y. Ootao (Osaka Prefecture University, Japan)

Y. Ozawa (Fukusima University, Japan)

P. C. Pandey (Indian Institute of Science, Bangalore, India)

Y. P. Shen (Xi' an Jiaotong University, China)

L. Tong (The University of Sydney, Australia)

W. Yang (Zhejiang University, China)

S. W. Yu (Tsinghua University, China)

T. Y. Zhang (The Hong Kong University of Science and Technology, China)

\section{International organizing committee}

T. Adachi (Toyohashi University of Technology, Japan)

Md. Afsar Ali (Bangladesh University of Engineering and Technology, Bangladesh)

H. G. Beom (Inha University, Korea)

W. Q. Chen (Zhejiang University, China)

T.-C. Chiu (National Cheng Kung University, Taiwan)

M.-H. Cho (Seoul National University, Korea)

C. F. Gao (Nanjing University of Aeronautics and Astronautics, China)

S. Gopalakrishnan (Indian Institute of Science, Bangalore, India)

T. Kant (Indian Institute of Technology Bombay, India)

N.-A. Noda (Kyushu Institute of Technology, Japan)

G. Prathap (National Institute of Science Communication and Information Resources, India)

Y. Shibutani (Osaka University, Japan)

V. P. W. Shim (National University of Singapore, Singapore)

M. Sunar (King Fahd University of Petroleum and Minerals, Saudi Arabia)

G. Sundararajan (International Advanced Research Center for Powder Metallurgy and New Materials, India)

J. Wang (Ningbo University, China)

Z. Zhong (Tongji University, China)

\section{National organizing committee}

Chair: Suhail Ahmad (Indian Institute of Technology Delhi, India)

\section{Members:}

D. Chakraborty (Indian Institute of Technology Guwahati)

R. Ganguly (Indian Institute of Science, Bangalore) 
K. Gupta (Indian Institute of Technology Delhi)

R. S. Jangid (Indian Institute of Technology Bombay)

C. Lakshman Rao (Indian Institute of Technology Mādras)

V. Menezes (Indian Institute of Technology Bombay)

S. Raja (National Aerospace Laboratories, Bangalore)

L. S. Ramachandra (Indian Institute of Technology Kharagpur)

M. C. Ray (Indian Institute of Technology Kharagpur)

K. K. Shukla (National Institute of Technology, Allahabad)

C. S. Upadhyay (Indian Institute of Technology Kanpur)

R. Velmurugan (Indian Institute of Technology Madras)

C. Venkatesan (Indian Institute of Technology Kanpur)

\section{Technical program committee}

Hon. chair:

K. Gupta (Indian Institute of Technology Delhi)

Chair:

S. Kapuria (Indian Institute of Technology Delhi)

\section{Members:}

Suresh Bhalla (Indian Institute of Technology Delhi)

P. Mahajan (Indian Institute of Technology Delhi)

V. Matsagar (Indian Institute of Technology Delhi)

S. V. Modak (Indian Institute of Technology Delhi)

B. P. Patel (Indian Institute of Technology Delhi)

S. Roy (Indian Institute of Technology Delhi)

S. P. Singh (Indian Institute of Technology Delhi)

M. K. Singha (Indian Institute of Technology Delhi)

S. V. Veeravalli (Indian Institute of Technology Delhi)

\section{Local organizing committee}

\section{Hon. Chair:}

S. N. Singh (Indian Institute of Technology Delhi)

\section{Chair:}

S. Ahmad (Indian Institute of Technology Delhi)

\section{Members:}

A. Ganguli (Indian Institute of Technology Delhi)

S. Hegde (Indian Institute of Technology Delhi)

S. Pradyumna (Indian Institute of Technology Delhi)

Rajesh Prasad (Indian Institute of Technology Delhi)

S. Sanghi (Indian Institute of Technology Delhi)

D. K. Sehgal (Indian Institute of Technology Delhi)

Balaji Srinivasan (Indian Institute of Technology Delhi)

Aravind K. Swamy (Indian Institute of Technology Delhi)

V. Tiwari (Indian Institute of Technology Delhi) 